\title{
Short-Term Cigarette Smoking in Rats Impairs Physical Capacity and Induces Cardiac Remodeling
}

\author{
Danilo Sales Bocalini (D, ${ }^{1}$ Rafael da Silva Luiz, ${ }^{2}$ Kleiton Augusto Santos Silva, ${ }^{3}$ \\ Andrey Jorge Serra, ${ }^{4}$ Renata Andrade Avila, ${ }^{5}$ André Soares Leopoldo, ${ }^{1}$ \\ Ana Paula Lima-Leopoldo, ${ }^{1}$ Márcia Regina Holanda da Cunha, ${ }^{1}$ Paulo Jose F. Tucci, ${ }^{4}$ \\ and Leonardo dos Santos ${ }^{5}$ \\ ${ }^{1}$ Experimental Physiology and Biochemistry Laboratory, Center of Physical Education and Sports, Federal University of \\ Espírito Santo, Vitoria, Brazil \\ ${ }^{2}$ Department of Medicine, Nephrology Division, Federal University of São Paulo, São Paulo, Brazil \\ ${ }^{3}$ Department of Biomedical Sciences, University of Missouri, Columbia, MO, USA \\ ${ }^{4}$ Department of Medicine, Cardiology Division, Federal University of São Paulo, São Paulo, Brazil \\ ${ }^{5}$ Department of Physiological Sciences, Center of Health Sciences, Federal University of Espirito Santo, Vitoria, Brazil
}

Correspondence should be addressed to Danilo Sales Bocalini; bocaliniht@hotmail.com

Received 17 June 2020; Revised 4 October 2020; Accepted 24 October 2020; Published 23 November 2020

Academic Editor: Toshiyuki Sawaguchi

Copyright ( 2020 Danilo Sales Bocalini et al. This is an open access article distributed under the Creative Commons Attribution License, which permits unrestricted use, distribution, and reproduction in any medium, provided the original work is properly cited.

\begin{abstract}
Despite the strong evidence on the cardiac and renal damages after chronic exposure to cigarette smoke, there is a paucity of data on its short-term effects. The study evaluated the short-term effects of cigarette smoking on left ventricular (LV) remodeling, in vitro myocardial and renal function. Female Wistar rats were randomized to control (C) and cigarette smoking rats for eight weeks. Physical capacity was assessed using an adapted model of exhaustive swim; left ventricle (LV) morphology and function were also evaluated. Renal function was assessed by creatinine clearance and urine protein. The in vitro myocardial performance was analyzed in isolated papillary muscles. Rats exhibited reduced physical capacity after short-term cigarette smoking. Although there was no change on LV function, reduced chamber diameter was found in the smoking group associated with an increased LV wall thickness. There was augmented cardiac mass compared to $\mathrm{C}$ that was confirmed by increased cardiomyocyte nucleus volume, but in vitro myocardial performance and renal function were unchanged. A short-term cigarette smoking induces cardiac remodeling without abnormalities in function. The smoking group still preserved renal function and in vitro myocardial performance. However, the reduced physical capacity may suggest an impairment of the cardiac reserve.
\end{abstract}

\section{Introduction}

Cigarette smoking is the leading cause of preventable morbidity and premature mortality at developed countries [1]. The main health disorders linked to smoking are cardiovascular diseases, cancer, and chronic obstructive pulmonary disease. In addition, smoking is an important risk factor to myocardial infarction [2] and nephropathies [3].

Studies have reported that a long-term cigarette smoke exposure may be associated with cardiac remodeling [4-6] which in turn correlates with myocardial dysfunction, heart failure, and increased mortality risk [7]. However, there are few studies $[8,9]$ that define the cardiotoxic effects of shortterm cigarette use, specifically reporting data from myocardial mechanics, which could indicate the initial and possibly reversible changes in the cardiocirculatory system. If concerning renal implications, although long-term exposure to cigarette smoke is an independent risk factor for microalbuminuria, diuresis and proteinuria $[10,11]$, the short-term effects on the renal function are still poorly understood as well. 
Thus, to date, a number of evidences have been reported the local and systemic effects of chronic exposure to cigarette smoke and such studies requiring prolonged exposure for several months. Therefore, the aim of this study was to evaluate the effects of short-term cigarette smoke exposure on myocardial function and remodeling and renal function and its consequences to the physical capacity of previously healthy rats.

\section{Materials and Methods}

Twenty female Wistar rats, weighing 200-230 g, were assigned to one of the following two groups: smoking animals $(n=10)$, exposed to cigarette smoke for eight weeks, and control animals $(n=10)$, allocated to the similar chamber but not exposed to tobacco smoke. The environment was controlled in terms of light (12 h light/dark cycle), clean-air room temperature $\left(23 \pm 3^{\circ} \mathrm{C}\right)$, and relative humidity $(60 \pm 5 \%)$. The study was conducted in accordance with the Basic \& Clinical Pharmacology \& Toxicology policy for experimental and clinical studies [12]. All procedures performed were in accordance with the Guide for the Care and Use of Laboratory Animals (NIH) and the ethical standards of the institution (Institutional Research Ethics Committee of the Federal University of São Paulo-protocol 34/08).

2.1. Exposure to Tobacco Smoke. Rats were exposed to cigarette smoke in a chamber (dimension: $1.000 \times 800 \times 700$ $\mathrm{mm}$ ) connected to a smoking device according to other studies $[5,6]$. The smoke was drawn out of filtered commercial cigarettes (composition per unit: $1.1 \mathrm{mg}$ nicotine, $14 \mathrm{mg}$ tar, and $15 \mathrm{mg}$ carbon monoxide) with a vacuum pump and exhausted into the smoking chamber. In the first week, cigarette number was gradually increased from 5 to 20 cigarettes for $60 \mathrm{~min}$, administered two times a day. Subsequently, 20 cigarettes were used in each smoking session, twice a day (60 min in the morning and $60 \mathrm{~min}$ in the afternoon) for eight weeks. The chamber carbon monoxide content was measured with a sensor Toxi Vision CO 860 (Biosystems, Prairieville, LA, USA).

2.2. Physical Capacity Swim Test. Physical capacity was assessed using an adapted model of exhaustive swimming previously reported [13]. A load equivalent to $10 \%$ of body weight was attached around the waist of each rat using a rubber band. Rats from all groups were observed to determine swimming time until exhaustion. Exhaustion was defined as the time-point when the rat could not swim up to the water surface for 10 seconds.

2.3. Renal Function. Renal function was measured after $8 \mathrm{wk}$ cigarette smoking exposure as previously described [14]. Briefly, rats were placed in a metabolic cage for $24 \mathrm{~h}$ with water and food ad libitum. After urine collection, samples were stored in $-20^{\circ} \mathrm{C}$. Serum (SCr, $\mathrm{mg} / \mathrm{dL}$ ) and urinary creatinine (UCr, $\mathrm{mg} / \mathrm{dL}$ ) were determined by Jaffé method (Creatinina K-Colorimetrico, Picratoalcalino, Labtest Diagnostica SA, Minas Gerais, Brazil). The creatinine clearance $(\mathrm{CrCl}, \mathrm{mL} / \mathrm{min})$ was calculated to estimate glomerular filtration rate. The urinary protein was measured by an enzymatic colorimetric assay (Sesiprot kit
Labtest Diagnostica SA, Minas Gerais, Brazil) and expressed as 24 -hour proteinuria (24h Uprot) or urine protein/creatinine clearance ratio (Uprot/CrCl).

2.4. Blood Pressure Measurement. Animals were allowed to adapt to the environment for three days before the measurement of blood pressure. Animals were placed in a heated chamber at a temperature of $38-40^{\circ} \mathrm{C}$ for ten minutes, and blood pressure values were recorded from each animal. At the end of protocols, mean, and systolic and diastolic blood pressures were determined by a tail cuff method (LETICA, LE5002, Barcelona, Spain) in conscious rats.

2.5. Doppler Echocardiography. At the end of protocols, rats were anesthetized with ketamine $(50 \mathrm{mg} / \mathrm{kg})$ plus xylazine $(10 \mathrm{mg} / \mathrm{kg})$ by i.p. route. Transthoracic echocardiographic was performed using a HP SONOS 5500 instrument (Philips Medical Systems, Andover, MA, USA), as described elsewhere $[15,16]$. The transverse images were obtained at basal (at the tip of the mitral valve leaflets), middle (at the papillary muscle level), and apical (distal to the papillary muscle but beyond the cavity cap) levels of the left ventricular (LV). The end-diastolic (LVEDD) and systolic (LVESD) LV diameters and diastolic (LVAWT) and systolic (LVPWT) LV posterior wall thicknesses were measured from the transverse parasternal view using M-mode images. The LV systolic function was defined by the fractional shortening (FS), and diastolic function was analyzed by the mitral diastolic influx velocity curve on the pulsed-wave Doppler. From the 4-chamber view, peak E-wave and A-wave velocities were acquired, and the E/A ratio was derived. Heart rate was calculated by a coupled electrocardiography.

2.6. In Vitro Myocardial Performance. Myocardial mechanic was evaluated as previously described $[17,18]$. LV posterior papillary muscles were carefully dissected and vertically mounted in an organ bath heated at $29^{\circ} \mathrm{C}$ and filled with an oxygenated Krebs-Henseleit buffered solution 100\% oxygenated and then attached to force transducer (Grass mod FT03E). Preparations were stimulated by platinum electrodes at frequency of $0.2 \mathrm{~Hz}$, using square-wave pulses of $5-\mathrm{ms}$ duration, and voltage adjusted to a value $10 \%$ greater than minimum required producing mechanical response. After 60 min equilibration period, the following parameters were evaluated: developed tension (DT), maximal rate of tension increase $(+\mathrm{d} T / \mathrm{d} t)$ and decrease $(-\mathrm{d} T / \mathrm{d} t)$, and resting tension (RT). At the end of the experiment, the muscle length was measured, and the muscular portion between the two clips was blotted dry and weighed. The cross-sectional area was estimated from the muscle weight and length by assuming a cylindrical shape and specific gravity of 1.0, and all forcerelated data were normalized by respective cross-sectional area and muscle mass.

2.7. Cardiac Biometry and Histomorphometry. Before the excision of the papillary muscle, atrium and ventricles were weighed separately, in order to use the chamber mass divided by the body mass as an index suggestive of hypertrophy. After papillary muscle dissection, ventricular samples were fixed in $10 \%$ buffered formalin and embedded in paraffin to 
evaluate nuclear volume and collagen content in histology slides $[6,16]$. Briefly, $7 \mu \mathrm{m}$ thickness sections were obtained from the LV and hematoxylin-eosin stained. Cardiac muscle fibers were visualized on a longitudinal axis using an Olympus microscope at 40x magnification, and ellipsoid nuclei were analyzed. As an estimative of myocyte hypertrophy, the average nuclear volume was determined randomly in 50 -70 myocytes cut longitudinally for each animal and calculated according to the following equation: nuclear volume $=$ $\pi \times D \times d 2 / 6$, where $d$ is the shorter nuclear diameter and $D$ is the longer nuclear diameter (15). LV collagen content was determined by picrosirius red staining using polarized light. The perivascular areas were excluded from analyzes. All histological images were visualized using an Olympus microscope at 5 randomized 40x magnification fields per animal and analyzed using Image Tool software 3.0.

2.8. Statistical Analysis. Analyses were performed using the SPSS (version 12.0, Chicago, Illinois, USA). All data are expressed as the mean \pm standard error of the mean (SEM). The D'Agostino-Pearson and Levene tests were used to verify approximately normal statistic distributions and variance homogeneity, respectively. Comparisons between groups were performed by unpaired Student's $t$ test or ANOVA two way conform necessary. Statistical significance was established at $p<0.05$.

\section{Results}

As showed at Figure 1, no differences were found in physical capacity before interventions (baseline time-point). However, rats exposed to cigarette smoke for eight weeks exhibited patent exhaustion in a shorter time (before: $197 \pm 7 v s$. after: $169 \pm 4$ seconds), but no changes were observed in control animals (before: $199 \pm 6$,after: $201 \pm 5$ seconds).

Despite the reduced physical capacity, no significant differences were found in systolic, diastolic, or mean blood pressures between the smoking and control groups (Table 1).

Additionally, Doppler echocardiography was performed in anesthetized rats to further evaluate cardiac morphology and function. Heart rate was significantly increased in the smoking group ( $243 \pm 28 v$ s. controls: $312 \pm 18 \mathrm{bpm})$, but without changes on LV fractional shortening and on the E/A ratio of the mitral valve flow study (Figure 2). Moreover, the group exposed to cigarette smoke significantly changed the cardiac morphology assessed by echocardiography: there was a reduction in LV diameters in both diastolic and systolic phases, associated with an increased LV posterior wall thickness compared with controls (Figure 2).

To further investigate if the unchanged cardiac global function in vivo was associated with preserved myocardial mechanics, the contractile response of papillary muscle was evaluated in vitro. As showed in Table 1, systolic (DT and + $\mathrm{d} T / \mathrm{d} t)$ and diastolic $(-\mathrm{d} T / \mathrm{d} t)$ performances of the isolated cardiac muscle were similar between groups.

Regarding renal function, as evaluated by urine protein levels and creatinine clearance, there were no statistically significant differences between the groups by short-term exposure to cigarette smoke (Table 1), although urinary

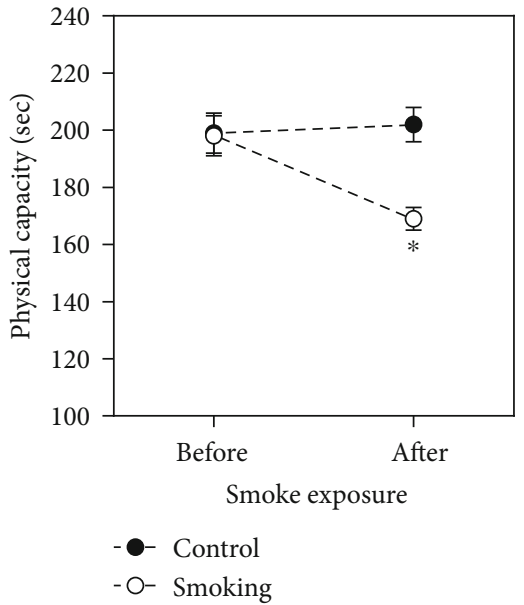

Figure 1: Physical capacity test performed by rats before and after smoke exposure in controls and smoking groups. Data expressed as mean $+/-$ SEM. ${ }^{*} p<0.05$ vs. before and control group.

TABle 1: Parameters of cardiac and renal functions of animals not exposed and exposed to cigarette smoke.

\begin{tabular}{lccc}
\hline & Control & Smoking & $p$ value \\
\hline Hemodynamic & & & \\
SBP $(\mathrm{mmHg})$ & $126 \pm 2$ & $129 \pm 2$ & $p>0.05$ \\
DBP $(\mathrm{mmHg})$ & $84 \pm 2$ & $82 \pm 3$ & $p>0.05$ \\
MBP $(\mathrm{mmHg})$ & $98 \pm 1$ & $98 \pm 2$ & $p>0.05$ \\
Myocardial function & & & \\
DT $\left(\mathrm{g} / \mathrm{mm}^{2} / \mathrm{mg}\right)$ & $1.11 \pm 0.57$ & $0.95 \pm 0.38$ & $p>0.05$ \\
$+\mathrm{d} T / \mathrm{d} t_{\text {max }}\left(\mathrm{g} / \mathrm{mm}^{2} / \mathrm{mg} / \mathrm{s}\right)$ & $10.13 \pm 3.57$ & $8.63 \pm 2.62$ & $p>0.05$ \\
$-\mathrm{d} T / \mathrm{d} t_{\mathrm{max}}\left(\mathrm{g} / \mathrm{mm}^{2} / \mathrm{mg} / \mathrm{s}\right)$ & $-8.18 \pm 5.88$ & $-5.43 \pm 1.82$ & $p>0.05$ \\
$\mathrm{RT}\left(\mathrm{g} / \mathrm{mm}^{2} / \mathrm{mg}\right)$ & $0.22 \pm 0.17$ & $0.19 \pm 0.06$ & $p>0.05$ \\
$\mathrm{Renal} \mathrm{function}$ & & & \\
$\mathrm{SCr}(\mathrm{mg} / \mathrm{dL})$ & $0.48 \pm 0.05$ & $0.47 \pm 0.08$ & $p>0.05$ \\
$\mathrm{CrCl}(\mathrm{mL} / \mathrm{min})$ & $1.14 \pm 0.02$ & $1.13 \pm 0.05$ & $p>0.05$ \\
Uprot $(\mathrm{mg} / 24 \mathrm{~h})$ & $16.27 \pm 1.60$ & $20.15 \pm 1.53$ & $p>0.05$ \\
Uprot$/ \mathrm{CrCl}(\mathrm{mg} / \mathrm{mL})$ & $14.78 \pm 1.40$ & $18.56 \pm 1.20$ & $p>0.05$ \\
\hline
\end{tabular}

Values are the mean \pm SEM. Systolic (SBP), diastolic (DBP), and mean blood pressures (MBP) measured in hemodynamic study; developed (DT) and resting tensions $(\mathrm{RT})$ and maximum rate of tension rise $\left(+\mathrm{d} T / \mathrm{d} t_{\max }\right)$ and decline (-dT/d $\left.t_{\max }\right)$ of isolated cardiac muscles; serum creatinine (SCr), creatinine clearance $(\mathrm{ClCr}), 24 \mathrm{~h}$ urinary excretion (Uprot), and urine protein/creatinine clearance ratio (Uprot/CrCl).

protein excretion indexed by creatinine clearance tended to increase in the smoking group $(p=0.0552)$.

Finally, the heart biometry and histomorphometry were also assessed (Figure 3), and smoking rats had increased LV mass and total cardiac mass compared with controls. Also, microscopic study indicated an increased nuclear volume of the LV cardiomyocytes from this group. In addition, myocardial interstitial collagen content was significant increased by short-term smoke exposure. 

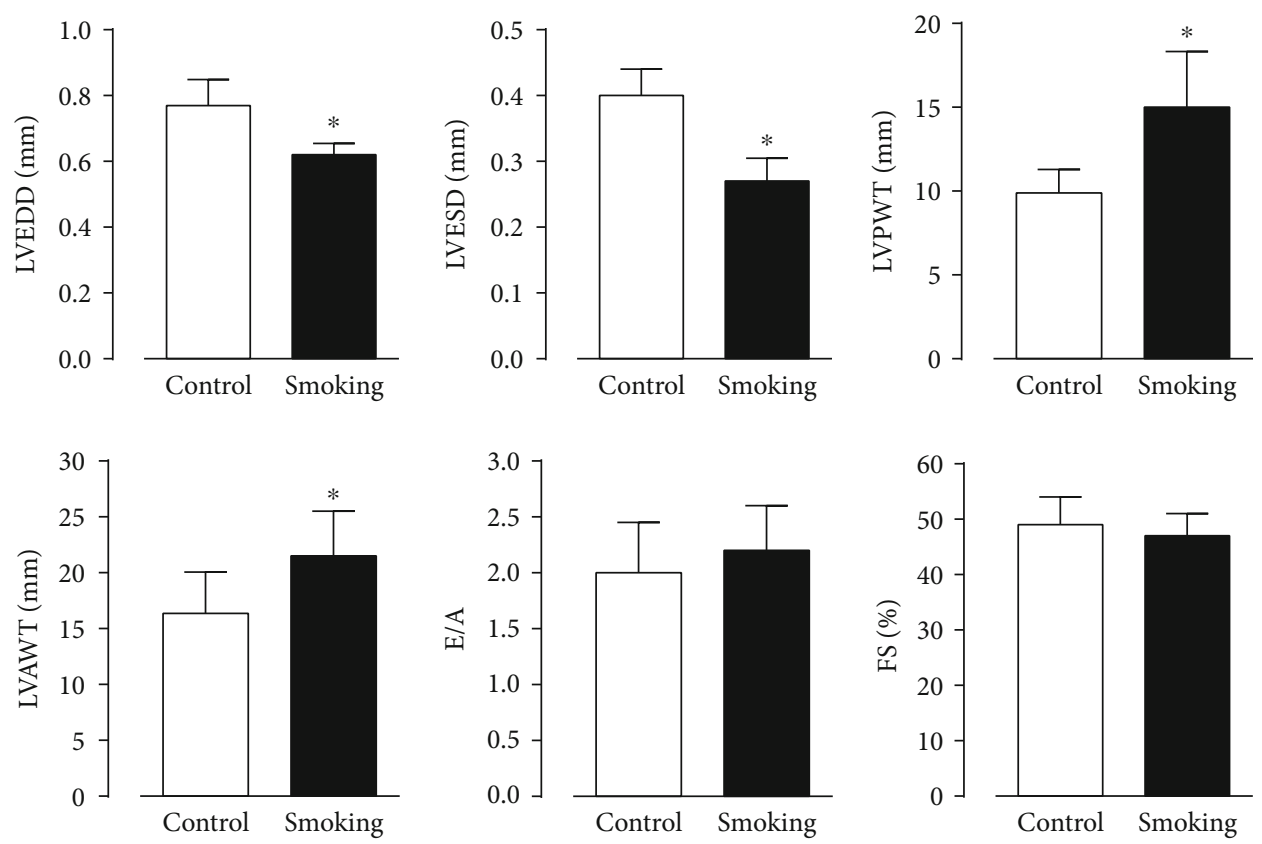

FIGURE 2: Morphofunctional data from Doppler echocardiography. Left ventricular end-diastolic (LVEDD) and end-systolic diameters (LVESD); LV posterior (LVPWT) and anterior wall thickness (LVAWT); relation between velocity of E and A waves (E/A ratio); and LV fractional shortening (FS). Bars are the means \pm SEM. ${ }^{*} p<0.05 v s$. control.

\section{Discussion}

The present study demonstrates that short-term exposure to cigarette smoke was capable of inducing an adverse cardiac remodeling associated with impaired physical capacity in previously healthy female rats. The level of carbon monoxide in the exposure chamber used in the present study was $\sim 510$ ppm, similar to reported by other authors using the same protocol $[5,6,19]$. According to these authors, an exposure to smoke from 40 cigarettes/day is sufficient to increase the rate of carboxyhemoglobin to $5.3 \pm 2.8 \%$ in rats, while animals that do not inhale smoke have $0.9 \pm 0.7 \%$ of carboxyhemoglobin on blood. It should be noted that values of carbon monoxide of $400 \mathrm{ppm}$ are sufficient to promote comparable to rates of carboxyhemoglobin found in humans considered heavy smokers [20]. Despite this, cardiac function evaluated in anesthetized animals and the myocardial performance evaluated in vitro still seems to be preserved after this short-term smoking.

Our data indicate that short-term exposure to smoke reduced the physical capacity of previously healthy rats. Actually, it is not possible to determine whether this physical capacity of smoking rats resulted from pulmonary, cardiovascular, or musculoskeletal abnormalities (i.e., skeletal muscle). However, it is noteworthy that the impairment of physical capacity is a well-known predictor of cardiovascular-related death [21] and that the improvement of the ability to perform exercise greatly contributes to reduce mortality due to cardiovascular disorders [22, 23]. Furthermore, exercise intolerance is a crucial point, because it directly affects self-related quality of life [24].

Regarding this, there are indications that chronic smoking impairs the physical capacity of the men $[25,26]$ and mitigates the tolerance to walking fatigue test [27]. Moreover, McDonough and Moffatt [28] stated that the chronically increased smoking-related blood carbon monoxide content could worsen exercise tolerance and reduce the maximal aerobic capacity, associated with an impaired glucose metabolism during exercise. An experimental study has also shown a reduction of $24 \%$ of aerobic capacity of animals exposed to carbon monoxide in similar dose to that found in individuals who smoke heavily [29]. For the authors, abnormalities in mitochondrial function in skeletal muscles can interfere with exercise capacity.

To the best of our knowledge, the impact of short-term cigarette smoking on the functional fitness and physical activity is few related by the literature. Regarding the effects of cigarette smoke on the cardiac function as a substrate for impaired physical capacity, there was proposed a possible association between smoking and functional fitness in heart failure [30]. However, given that both chronic smoking and heart failure syndrome could potentially reduce physical capacity not only by cardiac but also by peripheral changes such as pulmonary, hemodynamic, and neuro-motor functions, associated with water and salt kidney retention, our data indicates that short-term cigarette smoking may reduce functional fitness independent of heart or kidney failures.

Actually, there were no significant changes on GFR and urinary protein excretion in rats submitted to passive cigarette smoking for eight weeks, suggesting preserved glomerular and tubular functions. On the other hand, there are evidences that chronic cigarette smoking is associated with functional and structural renal changes in rats, demonstrating abnormal glomerular morphology after 16 weeks [11] and advanced hydropic degeneration of kidney after 24 weeks [31]. 


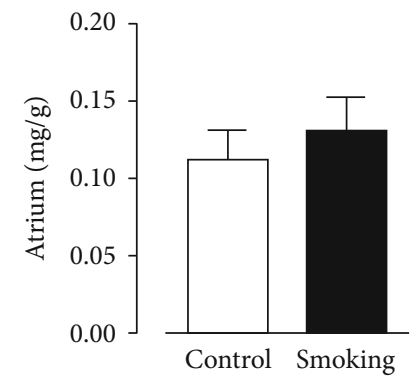

(a)
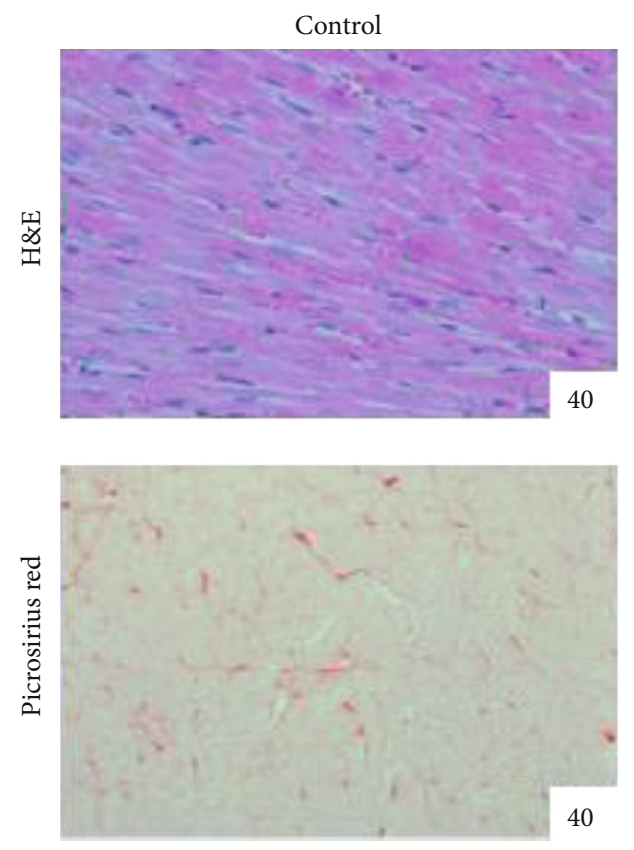

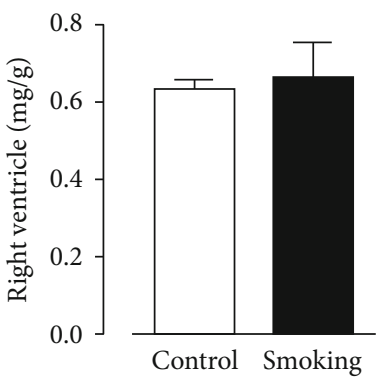

(b)

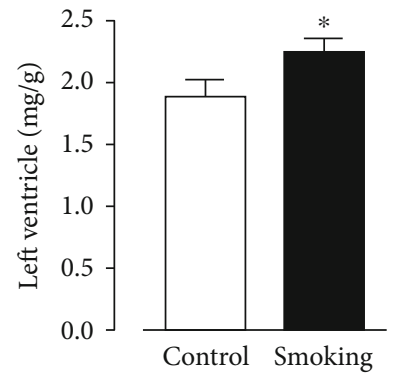

(c)

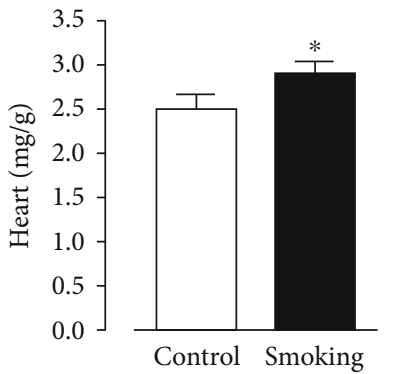

(d)
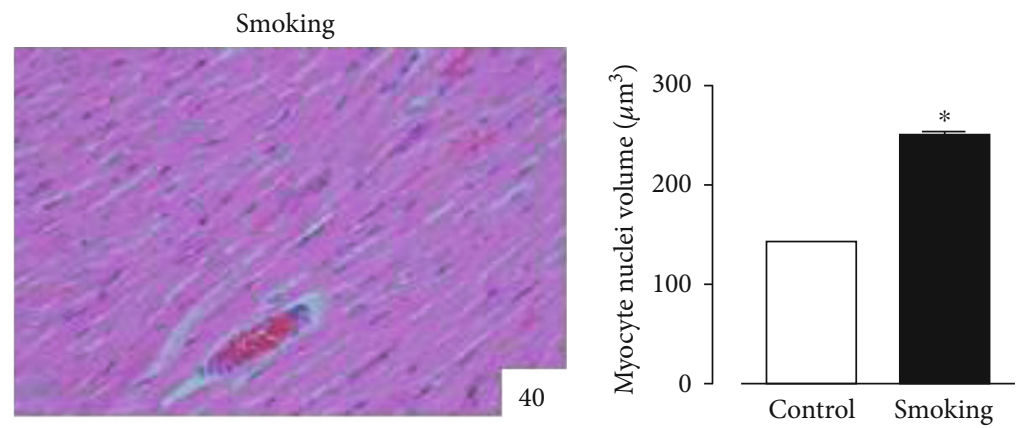

(e)

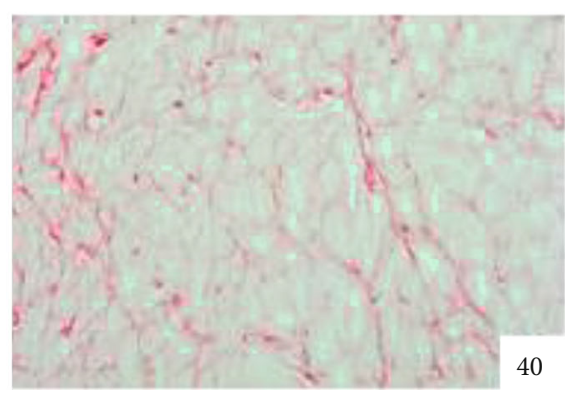

(f)

FIGURE 3: Cardiac biometry and histomorphometry. Atrial (a), right ventricular (b), left ventricular (c), and heart (d) masses were indexed to body mass. In the lower panels, representative microphotographies of the myocardium stained with hematoxylin-eosin (H\&E) for myocyte nuclei analysis and picrosirius red for interstitial collagen evaluation. Remodeling parameters including myocyte hypertrophy (e) and interstitial fibrosis (f) are graphically demonstrated. Bars are the means \pm SEM. ${ }^{*} p<0.05 v s$. control.

Conversely to previous observed in chronic smoke exposure $[5,6]$, but similar to described for short-term protocols $[8,9]$, echocardiographic data did not show ventricular systolic or diastolic dysfunction after this 8-week protocol, and no changes on blood pressure were noted as well. Moreover, cardiac muscle mechanics assessed in vitro was not affected, similar to also reported by Paiva et al. [19] after 4 weeks and Brooks et al. [32] after 16 weeks of smoking exposure in rats. Together, these findings could suggest that our experimental model of a short-term cigarette smoke exposure was not accompanied by cardiac dysfunction. However, about this apparent normality it is important noting that, under anesthesia or without substantial hemodynamic/mechanical loads, cardiac performance may appear unaltered, making the appropriate characterization of dysfunction difficult [15]. In spite of this supposed normality, when the hearts of smoking rats were submitted to a stress condition such as the physical capacity swim test, a significant degree of impairment was evident. Although we did not perform hemodynamic stress test and considering that noncardiac effects of smoking may also be present, we could just hypothesized that impaired physical capacity was due, at least in part, to a reduced cardiac reserve.

Notwithstanding, a previous study demonstrated that short-term of cigarette smoke induced cardiac morphological changes in rats [6] that could also be related to the reduced physical capacity. In fact, the increase in heart mass and myocyte nuclear volume and the reduced ventricular diameter after short-term cigarette smoking as suggestive of cardiac hypertrophy are not new data. There is no consensus about the hypertrophy mechanism involved in cardiac hypertrophy; however, the hypoxia caused carbon monoxide and nicotine. In the present study, the hypertrophic and fibrotic processes were not associated with changes on blood pressure, which suggests that this cardiac remodeling may be independent of hemodynamic loading. Regarding this, an important issue is the increased blood viscosity that could sustain increased cardiac afterload. Moreover, several other factors could act as additional mechanisms to trigger cardiac hypertrophic process during cigarette smoking: the generation 
of reactive oxygen species induced by smoking is knowingly cytotoxic to the myocardium [33]; nicotine has also been shown to be associated with myocardial remodeling $[34,35]$ and sympathetic hyperactivity [7], and although we did not assess sympathetic tone, heart rate was significantly elevated in the smoking group, which could play a role for the adverse cardiac remodeling; and evidence for the association between carbon monoxide/hypoxia and myocardial hypertrophy had also been showed $[29,36]$. Although we did not measure plasma levels of nicotine and cotinine, it is important note that these parameters are commonly used to estimate smoking intensity. In the present work, we used the carbon monoxide content on the exposure chamber to estimate smoking intensity, similar to other studies $[5,6,19]$. Thus, it is possible to consider that the hypertrophic response was linked to multiple effects of cigarette smoking not only to nervous and cardiovascular systems, but also by neurohumoral mechanisms. For example, it is well known that smoking, or more specifically nicotine, activates the sympathoadrenal system and increases the synthesis and release of noradrenaline and adrenaline [7, $37,38]$, and stimulates the renin-angiotensin-aldosterone system [39].

In conclusion, our results indicate that short-term cigarette smoke exposure in rats induced a concentric LV hypertrophy accompanied by increased in interstitial collagen. In addition, despite the apparent normal cardiac and renal functions, the smoking group has a significant impairment of the physical capacity, which could be suggested a result, at least in part, of a reduced cardiac reserve as a damaging consequence of this short-term smoking. It is noteworthy that recurrence of short-term smoking may have summative effects and thus constitute the mechanism underlying the ultimate chronic effects. Therefore, our data about the short-term effect of cigarette smoking can reflect an initial change in the pathophysiological mechanisms of smoking-induced chronic harmfulness.

\section{Data Availability}

All data are available if necessary.

\section{Disclosure}

The funders had no role in study design, data collection and analysis, decision to publish, or preparation of the manuscript.

\section{Conflicts of Interest}

The authors declare that they have no conflict of interest.

\section{Acknowledgments}

This work was supported by grants from the Coordenação de Aperfeiçoamento de Pessoal de Nível Superior (CAPES-finance code 001); Conselho Nacional de Desenvolvimento Científico e Tecnológico (grant number 303077/2017-4 CNPq 2018-20); and Fundação de Amparo à Pesquisa do Espírito Santo (grant numbers 80707483/2017 and 84417625/2018).

\section{References}

[1] C. E. Bartecchi, T. D. Mackenzie, and R. W. Schrier, "The human costs of tobacco use," The New England Journal of Medicine, vol. 330, no. 13, pp. 907-912, 1994.

[2] D. M. Burns, "Epidemiology of smoking-induced cardiovascular disease," Progress in Cardiovascular Diseases, vol. 46, no. 1, pp. 11-29, 2003.

[3] A. J. Bleyer, L. R. Shemanski, G. L. Burke, K. J. Hansen, and R. G. Appel, "Tobacco, hypertension, and vascular disease: risk factors for renal functional decline in an older population," Kidney International, vol. 57, no. 5, pp. 2072-2079, 2000.

[4] M. O. Boluyt, D. G. Penney, F. J. Clubb Jr., and T. P. White, "Exposure of neonatal rats to carbon monoxide alters cardiac adaptation to aortic constriction," Journal of Applied Physiology, vol. 70, no. 6, pp. 2697-2702, 1991.

[5] E. Castardeli, S. A. Paiva, B. B. Matsubara et al., "Chronic cigarette smoke exposure results in cardiac remodeling and impaired ventricular function in rats," Arquivos Brasileiros de Cardiologia, vol. 84, no. 4, pp. 320-324, 2005.

[6] D. Reis Junior, E. L. Antonio, M. F. de Franco, H. A. de Oliveira, P. J. Tucci, and A. J. Serra, "Association of exercise training with tobacco smoking prevents fibrosis but has adverse impact on myocardial mechanics," Nicotine \& Tobacco Research, vol. 18, no. 12, pp. 2268-2272, 2016.

[7] D. Gabriel-Costa, "The pathophysiology of myocardial infarction-induced heart failure," Pathophysiology, vol. 25, no. 4, pp. 277-284, 2018.

[8] L. Gu, V. Pandey, D. L. Geenen, S. A. K. Chowdhury, and M. R. Piano, "Cigarette smoke-induced left ventricular remodelling is associated with activation of mitogen-activated protein kinases," European Journal of Heart Failure, vol. 10, no. 11, pp. 1057-1064, 2008.

[9] P. P. Santos, F. Oliveira, V. C. M. P. Ferreira et al., "The role of lipotoxicity in smoke cardiomyopathy," PLoS One, vol. 9, no. 12, article e113739, 2014.

[10] J. M. Halimi and A. Mimran, "Renal effects of smoking: potential mechanisms and perspectives," Nephrology Dialysis Transplantation, vol. 15, no. 7, pp. 938-940, 2000.

[11] M. Dündar, I. Kocak, and N. Culhaci, "Effects of long-term passive smoking on the diameter of glomeruli in rats: histopathological evaluation," Nephrology, vol. 9, no. 2, pp. 53-57, 2004.

[12] P. Tveden-Nyborg, T. K. Bergmann, and J. Lykkesfeldt, "Basic \& clinical pharmacology \& toxicology policy for experimental and clinical studies," Basic \& Clinical Pharmacology \& Toxicology, vol. 123, no. 3, pp. 233-235, 2018.

[13] D. S. Bocalini, E. V. Carvalho, A. F. de Sousa, R. F. Levy, and P. J. Tucci, "Exercise training-induced enhancement in myocardial mechanics is lost after 2 weeks of detraining in rats," European Journal of Applied Physiology, vol. 109, no. 5, pp. 909-914, 2010.

[14] R. da Silva Luiz, K. A. S. Silva, R. R. Rampaso et al., "Exercise attenuates renal dysfunction with preservation of myocardial function in chronic kidney disease," PLoS One, vol. 8, no. 2, article e55363, 2013.

[15] L. dos Santos, E. L. Antonio, A. F. Souza, and P. J. Tucci, "Use of afterload hemodynamic stress as a practical method for assessing cardiac performance in rats with heart failure," Canadian Journal of Physiology and Pharmacology, vol. 88, no. 7, pp. 724-732, 2010. 
[16] L. dos Santos, G. A. Gonçalves, A. P. Davel et al., "Cell therapy prevents structural, functional and molecular remodeling of remote non-infarcted myocardium," International Journal of Cardiology, vol. 168, no. 4, pp. 3829-3836, 2013.

[17] D. S. Bocalini and P. J. Tucci, "Developed force of papillary muscle: what index correctly indicates contractile capacity?," International Heart Journal, vol. 50, no. 5, pp. 643-652, 2009.

[18] R. A. Ávila, M. A. S. C. Silva, J. V. Peixoto, I. Kassouf-Silva, R. T. H. Fogaça, and L. Dos Santos, "Mechanisms involved in the in vitro contractile dysfunction induced by different concentrations of ferrous iron in the rat myocardium," Toxicology In Vitro, vol. 36, pp. 38-45, 2016.

[19] S. A. de Paiva, L. A. Zornoff, M. P. Okoshi, K. Okoshi, A. C. Cicogna, and A. O. Campana, "Behavior of cardiac variables in animals exposed to cigarette smoke," Arquivos Brasileiros de Cardiologia, vol. 81, no. 3, pp. 221-228, 2003.

[20] R. F. Coburn, R. E. Forster, and P. B. Kane, "Considerations of the physiological variables that determine the blood carboxyhemoglobin concentration in man," The Journal of Clinical Investigation, vol. 44, no. 11, pp. 1899-1910, 1965.

[21] D. C. Lee, E. G. Artero, X. Sui, and S. N. Blair, "Mortality trends in the general population: the importance of cardiorespiratory fitness," Journal of Psychopharmacology, vol. 24, 4 suppl, pp. 27-35, 2010.

[22] F. Al-Khalili, I. Janszky, A. Andersson, B. Svane, and K. Schenck-Gustafsson, "Physical activity and exercise performance predict long-term prognosis in middle-aged women surviving acute coronary syndrome," Journal of Internal Medicine, vol. 261, no. 2, pp. 178-187, 2007.

[23] C. Hung, B. Daub, B. Black, R. Welsh, A. Quinney, and M. Haykowsky, "Exercise training improves overall physical fitness and quality of life in older women with coronary artery disease," Chest, vol. 126, no. 4, pp. 1026-1031, 2004.

[24] D. S. Bocalini, L. dos Santos, and A. J. Serra, "Physical exercise improves the functional capacity and quality of life in patients with heart failure," Clinics, vol. 63, no. 4, pp. 437-442, 2008.

[25] Y. Fukuba, N. Takamoto, K. Kushima et al., "Cigarette smoking and physical fitness," The Annals of Physiological Anthropology, vol. 12, no. 4, pp. 195-212, 1993.

[26] C. M. Bernaards, J. W. Twisk, W. Van Mechelen, J. Snel, and H. C. Kemper, "A longitudinal study on smoking in relationship to fitness and heart rate response," Medicine and Science in Sports and Exercise, vol. 35, no. 5, pp. 793-800, 2003.

[27] C. M. Trisltz, G. Ruas, L. K. Jamami, M. Jamani, and V. F. Couto, "Evaluation of the tolerance to the effort in smoking individuals," Fisioterapia em Movimento, vol. 20, pp. 55-61, 2017.

[28] P. McDonough and R. J. Moffatt, "Smoking-induced elevations in blood carboxyhaemoglobin Levels," Sports Medicine, vol. 27, no. 5, pp. 275-283, 1999.

[29] A. Bye, S. Sørhaug, M. Ceci et al., "Carbon monoxide levels experienced by heavy smokers impair aerobic capacity and cardiac contractility and induce pathological hypertrophy," Inhalation Toxicology, vol. 20, no. 7, pp. 635-646, 2008.

[30] S. J. Jay, "Passive smoking and the 6-minute walk test in heart failure," Chest, vol. 112, no. 1, pp. 289-290, 1997.

[31] Y. Cigremis, Y. Turkoz, M. Akgoz, and M. Sozmen, "The effects of chronic exposure to ethanol and cigarette smoke on the level of reduced glutathione and malondialdehyde in rat kidney," Urological Research, vol. 32, no. 3, pp. 213-218, 2004.
[32] W. W. Brooks, O. H. Bing, G. L. Huber, and W. H. Abelmann, "Contractile performance of rat myocardium after chronic tobacco smoke inhalation," Archives of Environmental Health, vol. 37, no. 2, pp. 93-97, 1982.

[33] Y. Sun, "Myocardial repair/remodelling following infarction: roles of local factors," Cardiovascular Research, vol. 81, no. 3, pp. 482-490, 2009.

[34] J. P. Loennechen, V. Beisvag, I. Arbo et al., "Chronic carbon monoxide exposurein vivoinduces myocardial endothelin-1 expression and hypertrophy in rat," Pharmacology \& Toxicology, vol. 85, no. 6, pp. 192-197, 1999.

[35] K. Greenspan, R. E. Edmands, S. B. Knoebel, and C. Fisch, "Some effects of nicotine on cardiac automaticity, conduction, and inotropy," Archives of Internal Medicine, vol. 123, no. 6, pp. 707-712, 1969.

[36] D. G. Penney and L. B. Bugaisky, "Non-coordinate expression of collagen mRNAs during carbon monoxide-induced cardiac hypertrophy Mol," Cell Biochemistry, vol. 109, pp. 37-41, 1992.

[37] G. Grassi, G. Seravalle, D. A. Calhoun et al., "Mechanisms responsible for sympathetic activation by cigarette smoking in humans," Circulation, vol. 90, no. 1, pp. 248-253, 1994.

[38] G. Grassi, G. Seravalle, D. A. Calhoun, G. Bolla, and G. Mancia, "Cigarette smoking and the adrenergic nervous system," Clinical and Experimental Hypertension. Part A, vol. 14, no. 1-2, pp. 251-260, 1992.

[39] Y. M. Yuan, L. Luo, Z. Guo, M. Yang, R. S. Ye, and C. Luo, "Activation of renin-angiotensin-aldosterone system (RAAS) in the lung of smoking-induced pulmonary arterial hypertension (PAH) rats," Journal of the Renin-AngiotensinAldosterone System, vol. 16, no. 2, pp. 249-253, 2015. 